\title{
Quantitation of SR 27417 in Human Plasma Using Electrospray Liquid Chromatography-Tandem Mass Spectrometry: A Study of Ion Suppression
}

\author{
Deborah L. Buhrman, Piper I. Price, and Patrick J. Rudewicz* \\ Sanofi Winthrop, Malvern, Pennsylvania, USA
}

\begin{abstract}
The effect of coeluting matrix compounds on the quantitation of SR 27417 in human plasma using electrospray liquid chromatography-tandem mass spectrometry has been examined. During the method development stage of this assay, plasma samples spiked with the analyte at $100 \mathrm{pg} / \mathrm{mL}$ were extracted using three different procedures: a hexane liquid-liquid extraction, an ethyl acetate back-extraction, and a solid phase extraction. Ion intensity of the analyte was found to be related inversely to the percent ionization of coeluting matrix components as evidenced by full scan spectra. The ethyl acetate back-extraction, which contained the fewest coeluting components, resulted in the highest ion intensity for the analyte. An assay comparison was done by using the liquid-liquid hexane and the ethyl acetate back-extractions for sample preparation. Replicate 1 -mL samples $(n=5)$ at 11 concentrations from 5 to $2000 \mathrm{pg} / \mathrm{mL}$ were extracted and analyzed. The results for the ethyl acetate back-extracted samples were acceptable from 2000 to $5 \mathrm{pg} / \mathrm{mL}$ with accuracy ranging from -11.6 to $2.61 \%$ of the nominal concentrations. In contrast, the hexane liquid-liquid method had poor accuracy and precision below $20 \mathrm{pg} / \mathrm{mL}$. The difference is explained by suppression of analyte ion intensity. These results are consistent with the current theory of electrospray ionization. (c) 1996 American Society for Mass Spectrometry (J Am Soc Mass Spectrom 1996, 7, 1099-1105)
\end{abstract}

$\mathrm{E}$ lectrospray liquid chromatography-tandem mass spectrometry (LC-MS/MS) is currently gaining widespread acceptance among pharmaceutical scientists for the quantitation of drugs and their metabolites in biological matrices. Although sensitivity in electrospray is compound dependent, excellent sensitivity has been reported for many drugs. For example, a minimum quantifiable level of $75 \mathrm{pg} / \mathrm{mL}$ was obtained for xanomeline, a muscarinic receptor antagonist [1]. Because very little heat is used in the ionization process, essentially no thermal degradation is observed for thermally labile compounds, which allows for the quantitative analysis of secondary metabolites such as glutathione, glucuronide, or sulfate conjugates. Indeed, the sensitivity for sulfate and glucuronide conjugates in the negative ion mode is excellent with a limit of detection of $10 \mathrm{pg} / \mathrm{mL}$ reported for sulfate conjugates of anabolic steroids [2].

Despite the success of electrospray for quantitative analysis, the technique does have certain limitations. One such fundamental problem is limited dynamic

Address reprint requests to Dr. Patrick J. Rudewicz, Allergan, 2525 Dupont Drive, P.O. Box 195534, Irvine, CA 92623-9534.

*Present address: Allergan, 2525 Dupont Drive, P.O. Box 19534, Irvine, CA $92623-9534$ range. Kebarle and co-workers [3,4] have reported linear responses from $10^{-8}$ to $10^{-5} \mathrm{M}$ for various organic bases. At about $10^{-5} \mathrm{M}$, the response no longer increases with concentration but levels off and eventually begins to decrease. The cause of the nonlinear response is under investigation by various research groups. Experiments by Bruins [5] indicate that the limited dynamic range is caused by an inability of droplet charge to be converted to gas-phase ions that can be mass analyzed.

Another problem with electrospray, at least in the area of quantitative analysis, is ion suppression. For example, Kebarle and co-workers [3] have shown that the electrospray responses, measured as $\mathrm{MH}^{+}$ions, of organic bases decrease with increasing concentration of another basic compound. The decrease in ion intensities of the $\mathrm{MH}^{+}$ions were, in some instances, attributed to gas-phase proton transfer reactions between the electrosprayed gas-phase ions and evaporated molecules of the stronger gas-phase base. However, solution phase chemistry was not ruled out.

Judging from these ion suppression studies [3] we thought that it would be reasonable to assume that similar results may be observed when quantitating a drug by using LC-MS/MS. Although not detected when only a product ion of the analyte is monitored, 
coeluting matrix components actually may reduce the ion intensity of the drug. Hence, ion suppression may adversely affect both the sensitivity and the reproducibility of a particular assay. We decided to test this premise during the development and validation of an electrospray LC-MS/MS method for the determination of SR 27417 (2-[ $N$-(2-dimethylaminoethyl)- $N$-(3pyridinylmethyl) amino]-4-(2,3,6 tri-isopropylphenyl) thiazole) (Figure 1)-a platelet-activating factor receptor antagonist-in human plasma.

\section{Experimental}

\section{Materials}

All chemicals were reagent grade or better. Control human plasma that contained ethylenediaminetetraacetic acid (EDTA) as the anticoagulant was obtained from Rockland (Gilbertsville, PA).

\section{Instrumentation}

Mass spectrometry parameters. The mass spectrometer used was a Sciex (Thornhill, Ontario) API III Plus with ion spray (pneumatically assisted electrospray) ionization. The ion spray voltage was $4400 \mathrm{~V}$, the orifice potential was $40 \mathrm{~V}$, the curtain gas was $1.2 \mathrm{~L} / \min \left(\mathrm{N}_{2}\right)$, the collision gas thickness was $210 \times 10^{12}$ molecules/ $\mathrm{cm}^{2}$ (argon), the nebulizer pressure was $50 \mathrm{lb} / \mathrm{in}^{2}$ (air), and the collision energy was $30 \mathrm{eV}$. The experiments were done in the positive ion mode. For the quantitative experiments, the mass spectrometer was scanned in the multiple reaction monitoring (MRM) mode in which the protonated molecule ion was collisionally dissociated and a fragment ion that corresponds to loss of the dimethylamino group was monitored (Figure 1). For SR 27417, this corresponds to $m / z$ $465 \rightarrow 420$. For the stable isotopically labeled internal standard $\left({ }^{2} \mathrm{H}_{4}-\mathrm{SR} 27417\right)$, four deuteriums are located on the pyridine ring and the corresponding MRM scan was $m / z 469 \rightarrow 424$. SR 27417 and the internal standard had retention times of approximately $3 \mathrm{~min}$ (Fig. 4).

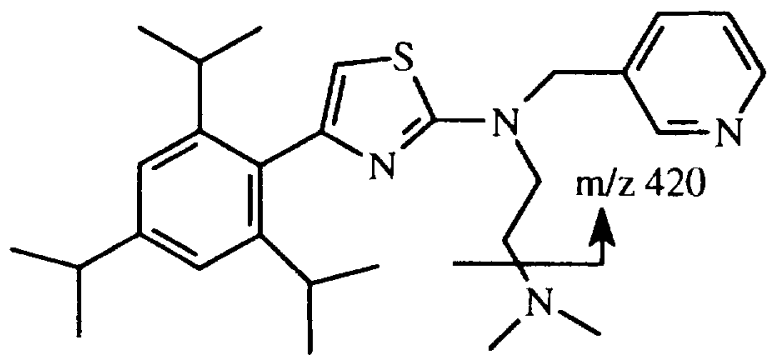

Figure 1. Structure of SR 27417, MW 464. The compound was quantitated in the multiple reaction monitoring mode: $\mathrm{m} / z 465$ $\left[\mathrm{MH}^{+}\right] \rightarrow m / z 420$.
Data were acquired by using the PE Nelson Sciex software RAD (version 2.4). The peak area ratios and linear regressions were generated by using the $\mathrm{PE}$ Nelson Sciex Software MacQuan (version 1.3).

HPLC parameters. A Shimadzu (Kyoto, Japan) highperformance liquid chromatography (HPLC) system that consisted of the following components was used: two LC-10AD pumps; SCL-10A controller; SIL-10A autosampler; CTO-10A column oven. HPLC column: Keystone BDS Hypersil C18 $(100 \times 2 \mathrm{~mm}) 5 \mu \mathrm{m}$. Column oven: $50^{\circ} \mathrm{C}$.

There were two mobile phases: $\mathrm{A}$-aqueous $2 \mathrm{mM}$ ammonium acetate: $0.2 \%$ formic acid; $\mathrm{B}-2 \mathrm{mM}$ ammonium acetate: $0.2 \%$ formic acid in acetonitrile. The flow rate was $200 \mu \mathrm{L} / \mathrm{min}$ with a total flow of 100 $\mu \mathrm{L} / \mathrm{min}$ to the mass spectrometer.

The HPLC gradient was as follows: At time $0 \mathrm{~min}$, $70 \% \mathrm{~B}$; B was increased from 70 to $100 \%$ in $1 \mathrm{~min}$; B was held at $100 \%$ for $2 \mathrm{~min}$; B was decreased to $70 \%$ in $1 \mathrm{~min}$; B was held at $70 \%$ for $2.2 \mathrm{~min}$. The run ended at $6.2 \mathrm{~min}$.

\section{Sample Processing}

Hexane liquid-liquid extraction. A $1 \mathrm{~mL}$ plasma sample was spiked with internal standard $(400 \mathrm{pg} / \mathrm{mL}$ for the comparison study and $800 \mathrm{pg} / \mathrm{mL}$ for the assay validation) and mixed with $500 \mu \mathrm{L}$ of $0.1-\mathrm{M}$ ammonium acetate adjusted to $\mathrm{pH} 6.0$. After vortex mixing, $6 \mathrm{~mL}$ of $n$-hexane was added and then rotary mixed for 20 $\mathrm{min}$. The sample was centrifuged for $5 \mathrm{~min}$ at 3000 rpm and the organic layer was transferred to a conical test tube. The sample was evaporated to dryness under $\mathrm{N}_{2}$ and then reconstituted in 200- $\mu \mathrm{L} 50: 50(\mathrm{v} / \mathrm{v})$ acetonitrile:water. A $100 \mu \mathrm{L}$ aliquot was injected onto the HPLC mass spectrometry system.

Liquid-liquid back extraction. One and one-half milliliters of $0.1-\mathrm{M}$ sodium carbonate and $7 \mathrm{~mL}$ of ethyl acetate were added to a $1 \mathrm{~mL}$ plasma sample. The sample was rotary mixed for $5 \mathrm{~min}$, then centrifuged for $5 \mathrm{~min}$ at $3000 \mathrm{rpm}$. The organic layer was removed and $1 \mathrm{~mL}$ of $0.025 \mathrm{M}$ sulfuric acid was added to it. The sample was then rotary mixed for $5 \mathrm{~min}$ and centrifuged for $5 \mathrm{~min}$ at $3000 \mathrm{rpm}$. The organic was removed and discarded. One and one-half milliliters of $0.1 \mathrm{M}$ sodium carbonate and $5 \mathrm{~mL}$ of ethyl acetate were added to the aqueous layer. The sample was again rotary mixed for $5 \mathrm{~min}$ and centrifuged for $5 \mathrm{~min}$ at $3000 \mathrm{rpm}$. The organic layer was removed and evaporated under $\mathrm{N}_{2}$ at $40^{\circ} \mathrm{C}$. The sample was recon-

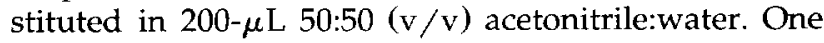
hundred $\mu \mathrm{L}$ was injected onto the HPLC column. This extraction procedure is a modified version of one previously used for an atmospheric pressure chemical ionization assay for abanoquil in blood [6]. 
Mixed mode solid phase extraction procedure. A $3 \mathrm{~mL}$ C18 Varian bond elute (Varian Associates, Sunnyvale, CA) was conditioned with $2 \mathrm{~mL}$ of saturated sodium chloride, then with $2 \mathrm{~mL}$ of $0.1 \mathrm{M}$ sodium phosphate buffer ( $\mathrm{pH}$ 6.0). One milliliter of plasma and $4 \mathrm{~mL}$ of $0.1 \mathrm{M}$ sodium phosphate buffer $(\mathrm{pH}$ 6.0) were vortex mixed, loaded to the column, and the liquid was removed by vacuum. The column was then washed with $1 \mathrm{~mL}$ of water and $1 \mathrm{~mL}$ of $20 \%$ acetonitrile in water. Next, $0.5 \mathrm{~mL}$ of $0.01 \mathrm{M}$ acetic acid was added to the column and the liquid was removed by vacuum. The column was then washed with $3 \mathrm{~mL}$ of methanol. The sample was eluted with $2 \mathrm{~mL}$ of $4 \%$ ammonium hydroxide in isopropanol:dichloromethane (27:75).

\section{Validation Experimental Design}

Preparation of calibration standards for validation. The calibration curve consisted of nine calibration levels. Calibration samples were prepared by spiking control human plasma $(5 \mathrm{~mL})$ with an aliquot $(25 \mu \mathrm{L})$ of SR 27417 to give nominal concentrations of 50,100, 200, $400,600,800,1000,1500$, and $2000 \mathrm{pg} / \mathrm{mL}$. The calibration standards were aliquoted into $1 \mathrm{~mL}$ volumes, processed by using hexane liquid-liquid extraction, and analyzed. Triplicate samples were run at the top $(2000 \mathrm{pg} / \mathrm{mL})$ and the bottom $(50 \mathrm{pg} / \mathrm{mL})$ concentrations. Each validation run included two plasma blanks with internal standard.

Preparation of quality control samples. Quality control samples were prepared in pools $(25$ or $50 \mathrm{~mL}$ ) by spiking control human plasma with an aliquot of SR 27417 to give nominal concentrations of 50, 100, 600, 1500 , and $2000 \mathrm{pg} / \mathrm{mL}$. The quality control samples were aliquoted into $1 \mathrm{~mL}$ volumes, processed by using hexane liquid-liquid extraction, and analyzed.

For the within-day study, replicate quality control samples $(n=6)$ were prepared at five concentrations $(50,100,600,1500$, and $2000 \mathrm{pg} / \mathrm{mL})$ and analyzed along with a full calibration curve (13 standard curve samples plus 2 plasma blanks). Quality control samples $(50,100,600,1500$, and $2000 \mathrm{pg} / \mathrm{mL}$ ) for the between-day runs were analyzed in duplicate on each day of five runs.

Treatment of data-Statistical analysis. The acceptance criteria for the within- and between-day validation studies were based on both the mean statistics determined for each quality control group and the deviation of the individual concentrations from their nominal values. For the lowest quality control level, the acceptance limit was $\pm 20 \%$, whereas for the remaining levels it was $\pm 15 \%$. Validation runs were accepted if the following criteria were met for each quality control level: the mean percent difference (eq 1, M\%D) was within $\pm 15 / 20 \%$; coefficient of variation (eq $2, C V$ ) was within $15 / 20 \%$; a minimum of $75 \%$ of all individ- ual samples were within $\pm 15 / 20 \%$ of their expected values with no more than two samples for any one group exceeding the appropriate limit.

$$
\begin{aligned}
& \mathrm{M} \% \mathrm{D}=\frac{(\text { observed conc. }- \text { nominal conc. })}{\text { nominal conc. }} \times 100 \\
& \mathrm{CV}=\frac{\text { standard deviation }}{\text { mean }} \times 100
\end{aligned}
$$

For the within-day validation, various weighting factors were investigated for the fitting of the calibration line. It was considered that $1 / Y^{2}$ gave the best fit to the calibration line and estimation of the true concentration of the quality control samples. This weighting was then used for subsequent between-day runs.

\section{Results and Discussion}

\section{Evaluation of Ion Suppression}

For analysis of an extracted plasma sample by electrospray ionization, the loss of signal for analyte may, in large part, be attributed to two processes: inefficient extraction efficiency and ion suppression. In an effort to evaluate these two processes independently, we performed the following experiments (A-C) using three different sample cleanup procedures: (1) liquid-liquid hexane extraction, (2) ethyl acetate backextraction, and (3) solid phase extraction. First, in experiment $A$, we measured the ion intensity of a $50 \mathrm{pg}$ standard of SR 27417 injected onto the HPLC column. For each of the three extraction procedures, we evaporated the extracted samples to dryness, reconstitutes in $200 \mu \mathrm{L}$ of solvent, and injected $100 \mu \mathrm{L}$ onto the LC-MS system. Hence, the signal observed from a $50 \mathrm{pg}$ neat standard sample represents what one would observe for a $100 \mathrm{pg} / \mathrm{mL}$ extracted sample if there were no loss of signal due to poor extraction or suppression of ion signal due to coeluting matrix components. Next, in experiment $\mathrm{B}$, we spiked a $1 \mathrm{~mL}$ plasma sample with $100 \mathrm{pg}$ of SR 27417, extracted the sample, and analyzed. The difference in ion intensity for SR 27417 between experiments $A$ and $B$ could be from either ion suppression or extraction losses. Experiment B is a measurement of the efficiency of the total process. Finally, in experiment $C$, we spiked a dried-down blank plasma extract with $200 \mu \mathrm{L}$ of a solution of SR $27417(0.5 \mathrm{pg} / \mu \mathrm{L})$, vortexed, and injected $100 \mu \mathrm{L}$ onto the HPLC column for analysis. Any loss in ion intensity for SR 27417 between experiments C and A would be due mostly to ion suppression. Extraction efficiency is determined by subtraction of the contribution of ion suppression from the process efficiency measured in experiment B. Each experiment was done in replicate $(n=5)$. The electrospray response for SR 27417 was measured as peak height.

Table 1 is a summary of the results of these three experiments for the three different extraction techniques. For the hexane liquid-liquid extraction evaluation, the mean peak height for five injections of SR 
27417 standards is 8580 . The mean obtained from extracted dried-down extracts that were spiked with SR 27417 (post-extraction, experiment C) is 6390. Assuming no differential loss due to adsorption, the ratio of these two numbers subtracted from 100 represents loss of signal due to ion suppression (100 - 100 (6390/ $8580)=26 \%$ ). Hence, $26 \%$ of the expected signal from a $100 \mathrm{pg} / \mathrm{mL}$ plasma sample extracted using a hexane liquid-liquid extraction will be lost due to ion suppression. The mean of five injections from spiked preextraction samples (experiment B) is 4050 . The ratio of $4050 / 8580$ corresponds to a total process efficiency of $47 \%$. Hence, $53 \%$ of the signal for SR 27417 is lost. If $26 \%$ of the total loss is due to ion suppression, $27 \%$ must be lost during the extraction procedure (extraction efficiency equal to $73 \%$ ).

For the ethyl acetate back-extraction, the extraction efficiency is similar to the hexane liquid-liquid extraction $(78 \%)$. However, with the back-extraction, ion suppression is essentially zero. Intuitively, this result is plausible because the back-extraction, which utilizes a $\mathrm{pH}$ change to extract into aqueous and then another $\mathrm{pH}$ change to extract back into organic, should result in a much cleaner extract than the simpler liquid-liquid hexane extraction. With no ion suppression, the total process efficiency of the back-extraction is simply equal to the extraction efficiency $(78 \%)$.

The results from the solid phase extraction procedure are also listed in Table 1 . The extraction efficiency is the same as that obtained for the back extraction (78\%). However, ion suppression for the solid phase extraction procedure is $41 \%$, which makes the overall process efficiency only $37 \%$. A summary of the results for the three extraction procedures is listed in Table 2.

If ion suppression is caused by matrix components that coelute with SR 27417 , we postulated that we should observe them in the full scan mode. To this end, we spiked blank plasma extracts with $200 \mu \mathrm{L}$ of a solution of SR $27417(0.25 \mathrm{ng} / \mu \mathrm{L})$, vortexed, and injected $100 \mu \mathrm{L}$ onto the HPLC column (25 ng of SR 27417 on column). We scanned the mass spectrometer from $\mathrm{m} / \mathrm{z} 200-700$ and summed spectra across the width of the SR 27417 chromatographic peak. The

Table 1. Evaluation of ion suppression by using three extraction techniques

\begin{tabular}{ccccc}
\hline & & $\begin{array}{c}\text { Neat } \\
\text { standard }\end{array}$ & $\begin{array}{c}\text { Spiked } \\
\text { pre-extraction }\end{array}$ & $\begin{array}{c}\text { Spiked } \\
\text { post-extraction }\end{array}$ \\
\hline \hline Hexane liq-liq Mean & $8580^{\mathrm{a}}$ & 4050 & 6390 \\
& CV & 9.38 & 13.0 & 6.29 \\
Back-extracted Mean & 8580 & 6680 & 9020 \\
\multirow{5}{*}{ Solid phase } & CV & 9.38 & 7.22 & 1.59 \\
& Mean & 10312 & 3800 & 6090 \\
& $C V$ & 6.4 & 18.1 & 16.5 \\
\hline
\end{tabular}

${ }^{a}$ Same injections. spectra for a neat standard of SR 27417 and the three spiked extracts are shown in Figure 2. The spectrum of the neat standard (Figure $2 \mathrm{a}$ ) contains a protonated molecule as the base peak at $m / z$ 465. The spectrum from the spiked back-extraction extract (Figure $2 b$ ) is very similar to that of the neat standard except for a peak at $m / z 520$, which presumably results from a coeluting matrix component. The spectrum from the spiked liquid-liquid hexane extraction (Figure 2c) also has the protonated molecule of SR 27417 as the base peak; however, major ions are present at $\mathrm{m} / z 241,374$, and 424 , which indicates that this extract is less clean than the back-extraction. The spectrum from the solid phase (Figure 2d) spiked extract contains matrix ions at $m / z 241,282,369,496,520$, and 546, among others. The protonated molecule ion of SR 27417 is no longer the largest peak in the spectrum.

At least qualitatively, there is a correlation between these spectra and ion suppression. The spectrum of the extract that shows the most ion suppression, derived from the solid phase extraction (41\% suppression), contains the largest percentage of ions that correspond to matrix components. The cleanest extract, derived from back-extraction, shows very little ion suppression and gives a spectrum with the smallest proportion of matrix ions. The liquid-liquid extract is intermediate between the two others in terms of both ion suppression $(26 \%)$ and percentage of ions in the spectrum that correspond to matrix components. If, as suggested elsewhere [3], proton transfer reactions occur in the high pressure region of the ion source between the protonated analyte and more basic matrix components, one would expect to see matrix ions in the resulting spectra. Of course, the possibility exists that other matrix components outside the scan range of $\mathrm{m} / \mathrm{z}$ 200-700 also may contribute to ion suppression.

From the experiments by Ikonomou, Blades, and Kebarle [3], suppression of ionization may occur when a coanalyte is present above concentrations of $10^{-5} \mathrm{M}$. At this concentration it was suggested that much of the coanalyte present in the electrospray droplets can evaporate as neutral molecules, assuming that the coanalyte is sufficiently volatile. If the gas-phase proton affinity of the coanalyte is higher than that of the analyte, proton transfer may occur, which decreases the ion intensity of the analyte.

From our experiments that used a $2 \mathrm{~mm}$ inner diameter HPLC column, the peak volume can be esti-

Table 2. Summary of ion suppression experiments

\begin{tabular}{lccc}
\hline & $\begin{array}{c}\text { Hexane } \\
\text { liquid-liquid }\end{array}$ & $\begin{array}{c}\text { Back- } \\
\text { extraction }\end{array}$ & $\begin{array}{c}\text { Solid phase } \\
\text { extraction }\end{array}$ \\
\hline Extraction efficiency & 73 & 78 & 78 \\
Ion suppression & 26 & 0 & 41 \\
Process efficiency & 47 & 78 & 37 \\
\hline
\end{tabular}



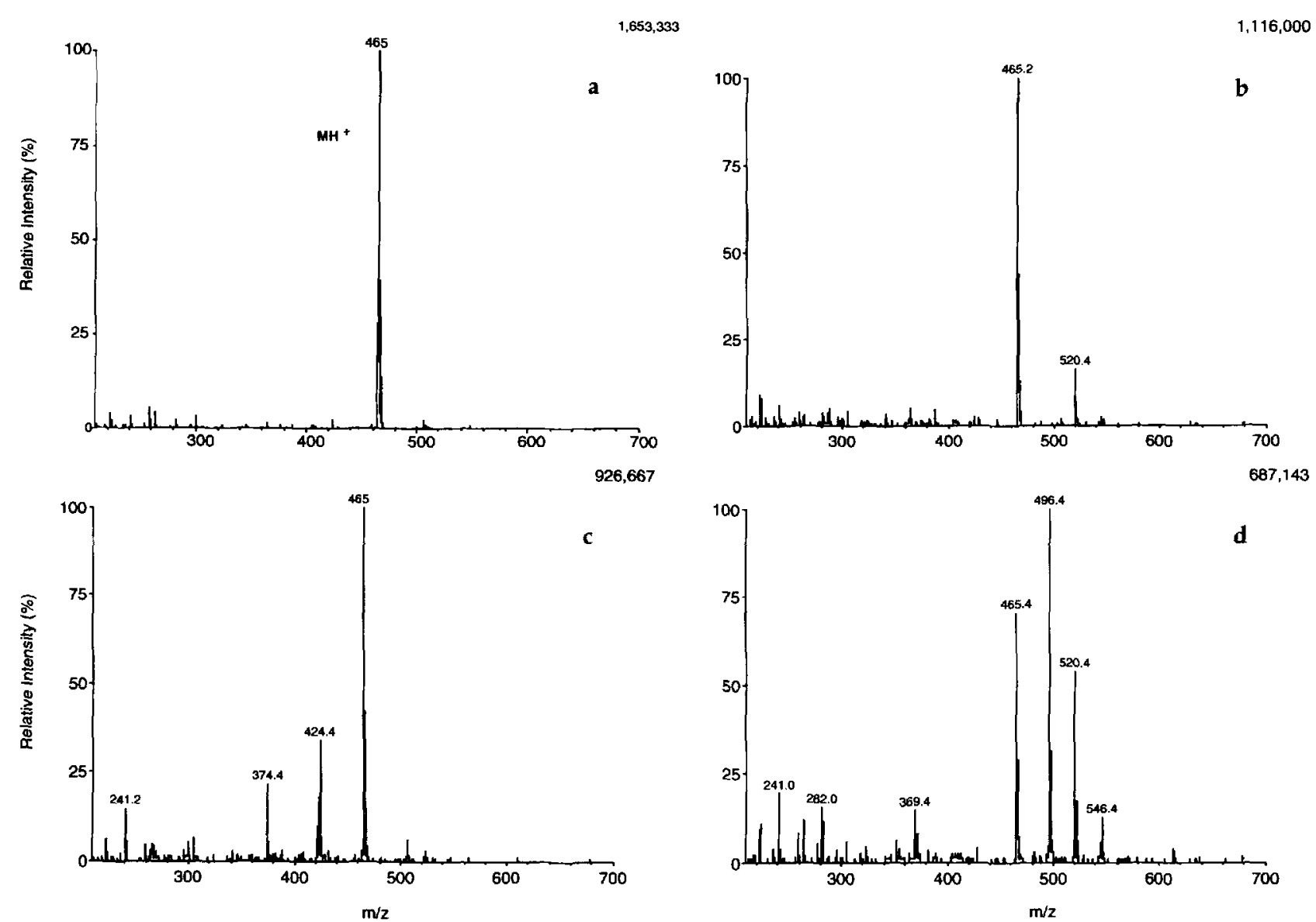

Figure 2. Full scan electrospray spectra of (a) SR 27417 (25 ng injected on-column); (b) $1 \mathrm{~mL}$ of blank human plasma extracted by using the ethyl acetate back-extraction and then spiked (post-extraction) with $25 \mathrm{ng}$ of SR 27417; (c) $1 \mathrm{~mL}$ of blank human plasma extracted by using the hexane liquid-liquid extraction and then spiked with $25 \mathrm{ng}$ of SR 27417; (d) $1 \mathrm{~mL}$ of blank human plasma extracted by using the solid phase extraction and then spiked with $25 \mathrm{ng}$ of SR 27417 .

mated by using the following equations [7]:

$$
\text { peak volume }=4 \sigma_{v(c o l)}
$$

and

$$
\sigma_{\mathrm{v}(\mathrm{col})}=\frac{V_{0} \times\left(1+k^{\prime}\right)}{N^{0.5}}
$$

where $\sigma_{\mathrm{v}(\mathrm{col})}$ is the volume standard deviation of the chromatographic peak, $V_{0}$ is the dead volume of the column, and $k^{\prime}$ is the capacity factor of the peak. Assuming that the typical plate count $(N)$ for this type of column is 6000 , the peak volume can be calculated to be approximately $25 \mu \mathrm{L}$ [7].

Therefore, assuming equal ionization efficiencies for SR 27417 and the coeluting matrix components, their concentrations can be estimated to be approximately 1 $\mathrm{ng} / \mu \mathrm{L}$ or between $10^{-6}$ and $10^{-5} \mathrm{M}$, which is near the concentration range that may cause ion suppression of the analyte.

Apart from gas-phase ion molecule chemistry, an alternate explanation for ion suppression exists. In the ion evaporation model of electrospray first outlined by Thomson and Iribarne [8], ions are emitted from droplets with radii on the order of $10 \mathrm{~nm}$ or less. At this size, the droplets are believed to contain a few tens of elementary charges and the surface field is $10^{9}$ $\mathrm{V} / \mathrm{m}$. If the samples are contaminated with involatile matrix components that prevent the droplets from reaching this critical radius and surface field, a reduction in ion signal for analyte would be expected [8].

\section{Preliminary Comparison of Different Extraction Methods}

As part of our method development, a preliminary comparison was done using the liquid-liquid hexane and the ethyl acetate back-extractions. Replicate 1-mL samples $(n=5)$ at 11 concentration levels from 5 to $2000 \mathrm{pg} / \mathrm{mL}$ were extracted and analyzed. The samples for both extraction procedures were aliquoted from the same plasma pools to eliminate the possibility of differences due to sample preparation. ${ }^{2} \mathrm{H}_{4}$-SR 27417 was used as an internal standard at $400 \mathrm{pg} / \mathrm{mL}$. 
Correlation Coeff, $=0.999$

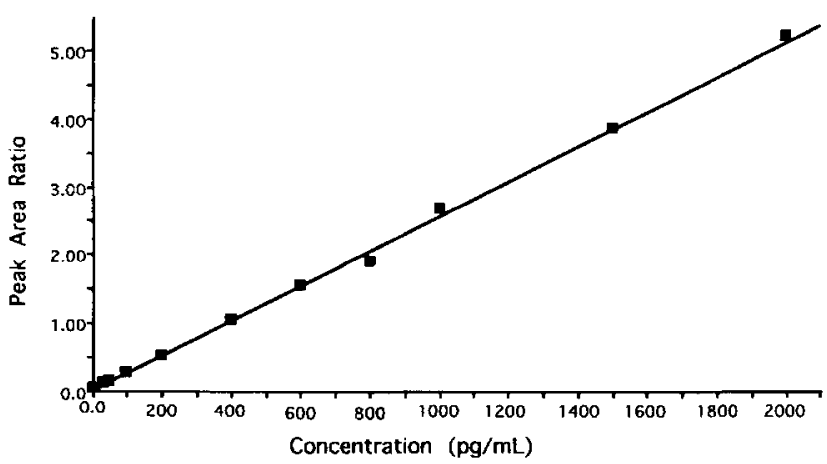

Figure 3. Calibration curve for the ethyl acetate back-extracted samples from 5 to $2000 \mathrm{pg} / \mathrm{mL}$.

The results from these experiments are listed in Table 3. For the hexane liquid-liquid extraction, the accuracy (mean percent difference, eq 1) and precision (percent coefficient of variation, eq 2) were acceptable from $2000 \mathrm{pg} / \mathrm{mL}$ down to $20 \mathrm{pg} / \mathrm{mL}$. In this range the accuracy ranged from -8.88 to $10.7 \%$ of nominal values with a precision ranging from 1.30 to $9.66 \%$. Below $20 \mathrm{pg} / \mathrm{mL}$, however, the accuracy and precision of the method were poor: at $5 \mathrm{pg} / \mathrm{mL}$ the accuracy and precision were 185 and $160 \%$, respectively. By comparison, the results for the ethyl acetate back-extraction were acceptable from 2000 to $5 \mathrm{pg} / \mathrm{mL}$ with accuracy that ranged from -11.6 to $2.61 \%$ of the nominal values and precision that ranged from 0.817 to $16.9 \%$. At 5 $\mathrm{pg} / \mathrm{mL}$ the accuracy and precision were -11.6 and $6.65 \%$, respectively. The calibration curve from 5 to $2000 \mathrm{pg} / \mathrm{mL}$ for the ethyl acetate back-extraction is shown in Figure 3.

From these results, it became evident that because the back-extraction samples were cleaner, the precision and accuracy below $20 \mathrm{pg} / \mathrm{mL}$ were better when compared to the liquid-liquid hexane extraction. We can relate this directly to the results of our ion suppression experiments and the full scan spectra shown in Figure 2. The more extensive sample cleanup results in fewer coeluting matrix components, which translates to less variability at lower levels and, in general, a lower limit of quantitation.

\section{Method Validation for SR 27417}

From the results described in the preceding text we realized that we probably could validate an analytical method to $20 \mathrm{pg} / \mathrm{mL}$ by using the liquid-liquid hexane extraction or perhaps lower with the liquid-liquid back-extraction. By using pharmacokinetic arguments, we decided that a minimum quantifiable limit of 50 $\mathrm{pg} / \mathrm{mL}$ would be sufficient to characterize the plasma concentration versus time profiles for our particular clinical study. It was then decided to use the less labor intensive hexane liquid-liquid extraction as opposed to the ethyl acetate back-extraction for sample processing.

A typical chromatogram at the minimum quantifiable concentration $(50 \mathrm{pg} / \mathrm{mL})$ from the assay validation is shown in Figure 4. The retention times of SR 27417 and ${ }^{2} \mathrm{H}_{4}$-SR 27417 were approximately $3 \mathrm{~min}$. The calibration curves were linear over the range of 50 to $2000 \mathrm{pg} / \mathrm{mL}$.

The accuracy $(\mathrm{M} \% \mathrm{D})$ of the within-day quality control samples ranged from -5.25 to $5.84 \%$ of the nominal values $(50,100,600,1500$, and $2000 \mathrm{pg} / \mathrm{mL}$ ). The precision $(\mathrm{CV})$ ranged between 0.840 and $6.93 \%$ (Table 4). The accuracy of the between-day quality control samples ranged from -1.57 to $11.5 \%$ of the nominal values with a precision between 2.31 and $12.3 \%$ (Table 5). Taking the minimum quantifiable level to be the lowest quality control concentration with precision and accuracy within $20 \%$, this assay was validated from 50 to $2000 \mathrm{pg} / \mathrm{mL}$ in human plasma.

Table 3. Method development comparison of two extraction procedures

\begin{tabular}{|c|c|c|c|c|c|c|}
\hline \multirow{2}{*}{$\begin{array}{l}\text { Nominal } \\
\text { conc. } \\
(\mathrm{pg} / \mathrm{mL})\end{array}$} & \multicolumn{3}{|c|}{ Ethyl acetate back-extraction } & \multicolumn{3}{|c|}{ Hexane liquid-liquid extraction } \\
\hline & Mean & $\mathrm{CV}$ & $M \% D^{a}$ & Mean & $\mathrm{CV}$ & $M \% D^{a}$ \\
\hline 5 & 4.42 & 6.65 & -11.6 & 14.2 & 160 & 185 \\
\hline 10 & 9.16 & 5.78 & -8.40 & 9.74 & 25.2 & -2.55 \\
\hline 20 & 19.8 & 5.16 & -0.946 & 19.9 & 4.17 & -0.523 \\
\hline 50 & 49.1 & 5.55 & -1.74 & 47.7 & 3.71 & -4.57 \\
\hline 100 & 103 & 3.94 & 2.61 & 100 & 2.10 & 0.766 \\
\hline 200 & 202 & 0.817 & 0.860 & 205 & 5.03 & 2.55 \\
\hline 400 & 402 & 1.52 & 1.57 & 410 & 1.90 & 2.62 \\
\hline 800 & 723 & 0.689 & -9.58 & 729 & 1.56 & -8.88 \\
\hline 1000 & 963 & 9.24 & -3.71 & 1110 & 9.66 & 10.7 \\
\hline 1500 & 1500 & 1.88 & 0.176 & 1530 & 1.30 & 2.13 \\
\hline 2000 & 1860 & 16.9 & -6.80 & 2040 & 1.36 & 1.83 \\
\hline
\end{tabular}

${ }^{\mathrm{a}} \mathrm{M} \% \mathrm{D}=[$ (observed conc. - nominal conc. $) /$ nominal conc. $] \times 100$ (eq 1$)$. 

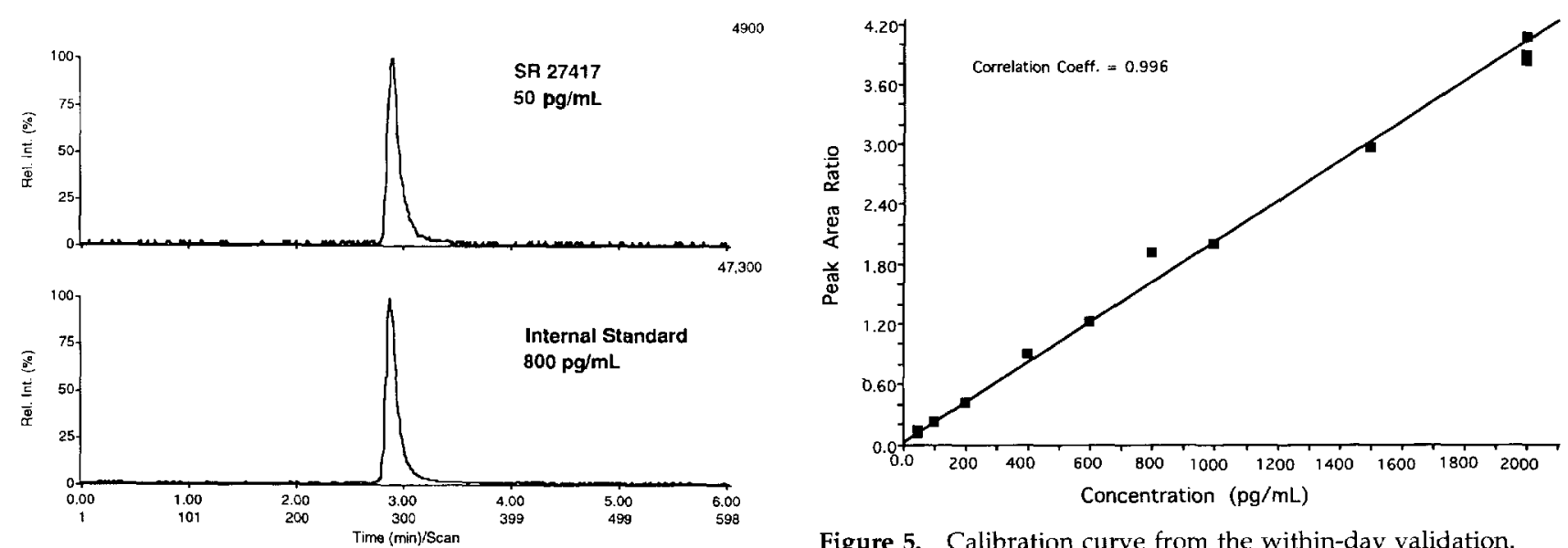

Figure 4. Typical $50 \mathrm{pg} / \mathrm{mL}$ sample from the assay validation of SR 27417 in human plasma.

Figure 5. Calibration curve from the within-day validation.

Table 4. Within-day validation data for the assay of SR 27417 in human plasma

\begin{tabular}{lccccc}
\hline & \multicolumn{5}{c}{ Nominal concentration $(\mathrm{pg} / \mathrm{mL})$} \\
\cline { 2 - 6 } & 50 & 100 & 600 & 1500 & 2000 \\
\hline \hline Mean & 52.9 & 104 & 623 & 1450 & 1900 \\
SD & 3.67 & 1.67 & 13.4 & 12.2 & 51.9 \\
CV & 6.93 & 1.60 & 2.15 & 0.840 & 2.74 \\
M\%D & 5.84 & 4.29 & 3.86 & -3.61 & -5.25 \\
\hline
\end{tabular}

Table 5. Between-day validation data for the assay of SR 27417 in human plasma

\begin{tabular}{lccccc}
\hline & \multicolumn{5}{c}{ Nominal concentration $(\mathrm{pg} / \mathrm{mL})$} \\
\cline { 2 - 6 } & 50 & 100 & 600 & 1500 & 2000 \\
\hline \hline Mean & 55.3 & 112 & 643 & 1490 & 1970 \\
SD & 1.87 & 13.7 & 14.9 & 39.4 & 50.2 \\
CV & 3.38 & 12.3 & 2.31 & 2.64 & 2.55 \\
M\%D & 10.7 & 11.5 & 7.21 & -0.43 & -1.57 \\
\hline
\end{tabular}

\section{Conclusions}

As electrospray becomes more accepted in bioanalytical laboratories for the quantitation of drugs in biological matrices, a more thorough understanding of the ionization process is necessary. In this study, the relationship between sample cleanliness and quantitative results as measured by assay accuracy and precision was explored. When tandem mass spectrometry is used for quantitation, the selectivity of multiple reaction monitoring often results in one peak, which corresponds to the analyte, in the chromatogram. Nevertheless, matrix components that are not detected but that coelute with the analyte may adversely affect the results of the assay through suppression of ionization. Elimination of these matrix components with better extraction methods may allow for quantitation at lower concentrations.

\section{References}

1. Murphy, A. T.; Bonate, P. L.; Kasper, S. C.; Gillespie, T. A.; DeLong, A. F. Biological Mass Spectrometry 1994, 23, 621-625.

2. Weidolf, L. O. G.; Lee, E. D.; Henion, J. D., Biomed. Environ. Mass Spectrom. 1988, 15, 283-289.

3. Ikonomou, M. G.; Blades, A. T.; Kebarle, P. Anal. Chem. 1990, 62, 957-967.

4. Kebarle, P.; Tang, L. Anal. Chem. 1993, 65, 972A-986A.

5. Bruins, A. P. Proceedings of the 7 th Sanibel Conference on Mass Spectrometry; Sanibel Island, FL, January 21-24, 1995.

6. Kaye, B.; Clark, M. W. H.; Cussans, N. J.; Macrae, P. V.; Stopher, D. A. Biological Mass Spectrometry, 1992, 21, 585-589.

7. Steiner, F. Applications of Narrow-Bore Columns in HPLC; Hewlett-Packard Company: Waldbronn, Germany, 1991.

8. Thomson, B. A.; Iribarne, J. V. J. Chem. Phys. 1979, 71, 4451-4463. 